\title{
NOTES ON THE DIOPHANTINE EQUATION $x^{2}+7 y^{2}=2^{n+2}$
}

\author{
S. B. TOWNES ${ }^{1}$
}

1. Introduction. In [1] the authors define $r=\frac{1}{2}\left[1+(-7)^{1 / 2}\right]$ and $r^{n}=\frac{1}{2}\left[b_{n-1}+a_{n-1}(-7)^{1 / 2}\right]$ with $b_{n-1}^{2}+7 a_{n-1}^{2}=2^{n+2}, n \geqq 1$. They prove that, except for $a_{0}=a_{1}=1$, and $a_{2}=a_{4}=a_{12}=-1,\left|a_{n}\right|>1$. They also prove that no integer appears in the sequence $\left\{a_{i}\right\}$ more than three times. In [2] Miss P. Chowla, using a different notation, proves that in the sequence $\left\{b_{i}\right\}$, except for $b_{0}=b_{3}=1$, an integer appears only once if $i+1$ is a power of 2 . She also states, without explicit proof, that no integer appears more than twice in the sequence $\left\{b_{i}\right\}$. In [1] the authors ask for an explicit formula $N(c)$ with the property that for an arbitrary positive integer $c$, if $n>N(c)$, then $\left|a_{n}\right| \neq c$.

It is convenient to change the notation so that $r^{n}=\frac{1}{2}\left[b_{n}+a_{n}(-7)^{1 / 2}\right]$, $b_{n}^{2}+7 a_{n}^{2}=2^{n+2}, n \geqq 1$, and $a_{1}=b_{1}=1$.

In these notes it will be shown that for $\left|a_{i}\right|>1$, no two terms of the sequence $\left\{a_{i}\right\}$ are equal, with the exception of $a_{4}=a_{8}=-3$. The desired formula $N(c)$ will be developed.

2. Proof of the uniqueness of the $a_{i}$. One may deduce from the definition of $r^{n}$ that

$$
2 a_{(n+1) s}=b_{n s} a_{s}+b_{s} a_{n s}
$$

and

$$
2 b_{(n+1) s}=b_{n s} b_{s}-7 a_{n s} a_{s} .
$$

Lemma 1. For all values of $n, a_{n}$ and $b_{n}$ are odd integers with $a_{n} \equiv b_{n}(\bmod 4)$.

The lemma is true for $a_{1}=b_{1}=1$. Assume it is true for arbitrary $n$. From (1) and (2), with $s=1,2 a_{n+1}=b_{n}+a_{n} \equiv 2(\bmod 4)$, and $2 b_{n+1}$ $=b_{n}-7 a_{n} \equiv 2(\bmod 4)$. Furthermore, $b_{n}+a_{n} \equiv b_{n}-7 a_{n}(\bmod 8)$, hence $a_{n+1} \equiv b_{n+1}(\bmod 4)$, which proves the lemma.

To get useful expressions for $b_{n s}$ and $a_{n s}$, expand

$$
\left[\frac{b_{s}+a_{s}(-7)^{1 / 2}}{2}\right]^{n}=\frac{b_{n s}+a_{n s}(-7)^{1 / 2}}{2}
$$

and get

Received by the editors August 31, 1961 and, in revised form, November 22, 1961.

1 The author thanks the referee for his suggestions, all of which have been incorporated in this paper. 


$$
2^{n-1} b_{n s}=b_{s}^{n}-\frac{7 n(n-1)}{2} b_{s}^{n-2} a_{s}^{2}+\cdots+A,
$$

in which $A$ is $n(-7)^{(n-1) / 2} a_{s}^{n-1} b_{s}$ if $n$ is odd, and $(-7)^{n / 2} a_{s}^{n}$, if $n$ is even. Also

$$
2^{n-1} a_{n s}=a_{s}\left[n b_{s}^{n-1}-\frac{7 n(n-1)(n-2)}{3 !} b_{s}^{n-3} a_{s}^{2}+\cdots+B\right],
$$

in which $B$ is $(-7)^{(n-1) / 2} a_{s}^{n-1}$ if $n$ is odd, and $(-7)^{(n-2) / 2} n a_{s}^{n-2} b_{s}$ if $n$ is even. Since $b_{s}^{2}+7 a_{s}^{2}=2^{s+2}$, one may substitute $2^{s+2}-b_{s}^{2}$ for $7 a_{s}^{2}$ in (3) and (4) and get, for all values of $n \geqq 2$,

$$
\begin{aligned}
& b_{n s}=b_{s}^{n}-n 2^{s} b_{s}^{n-2}+\frac{n}{2}\left(\begin{array}{c}
n-3 \\
1
\end{array}\right) 2^{2 s} b_{s}^{n-4}-\cdots \\
&+(-1)^{i-1} \frac{n}{i-1}\left(\begin{array}{c}
n-i \\
i-2
\end{array}\right) 2^{(i-1) s} b_{s}^{n-2 i+2}+\cdots \\
& 3 \leqq i \leqq \frac{1}{2}(n+2),
\end{aligned}
$$

and

$$
\begin{aligned}
& a_{n s}= a_{s}\left[b_{s}^{n-1}-(n-2) 2^{s} b_{s}^{n-3}+\cdots\right. \\
&\left.-(-1)^{i-1}\left(\begin{array}{c}
n-i \\
i-1
\end{array}\right) 2^{(i-1) s} b_{s}^{n-2 i+1}+\cdots\right] \\
& 2 \leqq i \leqq \frac{1}{2}(n+1) .
\end{aligned}
$$

One may verify (5) for $n=2$ and $n=3$ by actual computation. Now assume it is true for arbitrary $n \geqq 3$, and use (1) and (2) to get $2 b_{(n+1) s}$. Replace $-7 a_{s}^{2}$, which occurs in $-7 a_{n s} a_{s}$ in (6), by $b_{s}^{2}-2^{s+2}$. The first two terms of $2 b_{(n+1) s}$ are easily computed and agree with (5). The $i$ th term, which may be seen to use the $(i-1)$ st term of $(6)$, as well as the $i$ th terms of (5) and (6), is equal to

$$
(-1)^{i-1}\left[4\left(\begin{array}{c}
n+1-i \\
i-2
\end{array}\right)+\left(\begin{array}{c}
n-i \\
i-1
\end{array}\right)+\frac{n}{i-1}\left(\begin{array}{c}
n-i \\
i-2
\end{array}\right)\right] 2^{(i-1) s} b^{n-2 i+3},
$$

which, on simplifying, becomes

$$
(-1)^{i-1} \frac{2(n+1)}{i-1}\left(\begin{array}{c}
n+i-1 \\
i-2
\end{array}\right) 2^{(i-1) s} b^{n-2 i+3}
$$


and on division by 2 , becomes the $i$ th term of $b_{(n+1) s}$.

In a similar way (6) may be verified by mathematical induction.

Lemma 2. For $n$ odd and greater than $1, b_{n} \equiv 3(\bmod 8)$.

In the $i$ th term of (5), the coefficient of $2^{(i-1) s} b_{s}^{n-2 i+2}$ is easily seen to be an integer, since it is

$$
(-1)^{i-1}\left\{\left(\begin{array}{c}
n-i+1 \\
i-1
\end{array}\right)+\left(\begin{array}{c}
n-i \\
i-2
\end{array}\right)\right\} \text {. }
$$

Thus all terms after the third are multiples of 8 . Let $s=1$, and use (5) to see that $b_{n} \equiv 1-2 n+2 n(n-3) \equiv 1+2 n^{2} \equiv 3(\bmod 8)$, since $n$ is odd.

From here on, to avoid repetition, let the equation $x \equiv 1\left(\bmod 2^{t}\right)$ imply that $x \neq 1\left(\bmod 2^{t+1}\right)$.

LEMmA 3. For $n=2^{r}, r \geqq 3, b_{n} \equiv 1\left(\bmod 2^{r+2}\right)$.

Since $b_{8}=-31 \equiv 1\left(\bmod 2^{5}\right)$, the lemma is true for $n=3$. Assume the lemma true for arbitrary $r$ with $n=2^{r}, r \geqq 3$. Then by (5)

$$
b_{2 n}=b_{n}^{2}-2^{n+1}
$$

and

$$
b_{2 n} \equiv b_{n}^{2} \equiv 1\left(\bmod 2^{r+3}\right) \text {. }
$$

LemmA 4. With $n$ odd and greater than 1 , and $s=2^{r}, r \geqq 1, b_{n s}$ $\equiv 1\left(\bmod 2^{r+2}\right)$.

For $s=2$, use $(7)$ to see that $b_{2 n} \equiv 1\left(\bmod 2^{3}\right)$. For $s=4$, use $(7)$ again, with $n$ replaced by $2 n$ to get $b_{4 n} \equiv 1\left(\bmod 2^{4}\right)$. With $r \geqq 3$, so that $2^{r}>r+2$, use (5) to get $b_{n s}=b_{s}^{n}-n 2^{s} b_{s}^{n-2}+\cdots$. Since $b_{s}$ $\equiv 1\left(\bmod 2^{r+2}\right)$, and $n$ is odd, then $b_{n s} \equiv b_{s}^{n} \equiv 1\left(\bmod 2^{r+2}\right)$.

LeмmA 5. Except for $b_{1}=b_{4}=1,\left|b_{n}\right|>1$.

The values of $b_{1}$ and $b_{4}$ are easily computed. The remainder of the lemma follows from Lemmas 2, 3, and 4 .

Lemma 6. For $m$ any odd integer, and $a_{r} \equiv 0(\bmod m)$, then $a_{r+s} \equiv 0$ or $\not \equiv 0(\bmod m)$ according as $a_{s} \equiv$ or $\not \equiv 0(\bmod m)$.

Since $\left(a_{r}, b_{r}\right)=\left(a_{s}, b_{s}\right)=1$, and $2 a_{r+s}=a_{r} b_{s}+a_{s} b_{r} \equiv a_{s} b_{r}(\bmod m)$, the lemma is true.

LEMмA 7. If $t$ is the least value of $n$ for which $a_{n} \equiv 0(\bmod m), m$ any odd integer greater than 1 , then $a_{u} \equiv 0(\bmod m)$ if and only if $u$ is $a$ multiple of $t$.

If $u$ is a multiple of $t$, then $a_{u} \equiv 0(\bmod m)$ by $(6)$. To prove the con- 
verse let $u=q t+v, 0 \leqq v<t$. Then $2 a_{u}=a_{q t} b_{v}+a_{v} b_{q t} \equiv a_{v} b_{q t}(\bmod m)$. Now $\left(a_{n}, b_{n}\right)=1$, and if $0<v<t$ then $a_{n} \neq \equiv 0(\bmod m)$. Hence $v=0$ and the lemma follows.

LEMMA 8. For $n=1$ and for $n=4, a_{2 n}=a_{n}$, but for all other values of $n,\left|a_{2 n}\right|>\left|a_{n}\right|$.

In general, $a_{2 n}=a_{n} b_{n}$. But $b_{1}=b_{4}=1$, and for all other values of $n$, by Lemma $5,\left|b_{n}\right|>1$.

Lemma 9. For $s>1$, and $n=3,5$, or 13 , then $\left|a_{n s}\right|>\left|a_{s}\right|$.

For $s=2,3$, or 4 , the lemma may be verified by actually computing $a_{n s}$ and $a_{s}$ in each case. Now assume $s \geqq 5$, and by (6)

$$
\begin{aligned}
& a_{3 s}=a_{s}\left(b_{s}^{2}-2^{s}\right), \\
& a_{5 s}=a_{s}\left(b_{s}^{4}-3 \cdot 2^{s} b_{s}^{2}+2^{2 s}\right), \\
& a_{13 s}=a_{s}\left(b_{s}^{12}-11 \cdot 2^{s} b_{s}^{10}+\cdots\right) .
\end{aligned}
$$

Since the proofs are closely analogous, only the first will be given in detail. In each case, the proof is accomplished by showing the coefficient of $a_{s}$ is greater than 1 in absolute value by showing it is $\not \equiv 1\left(\bmod 2^{s}\right)$ but $\equiv 1(\bmod 8)$. To prove that $\left|a_{3 s}\right|>\left|a_{s}\right|$, note that if $s$ is odd then $b_{s} \equiv 3(\bmod 8)$, and therefore $b_{s}^{2} \equiv 1(\bmod 8)$, but $\not \equiv 1\left(\bmod 2^{s}\right)$. If $s$ is even, let $s=k \cdot 2^{r}$, with $k$ odd. By Lemma 4, $b_{s}=q \cdot 2^{r+2}+1, q$ odd, and therefore $b_{s}^{2} \equiv 1\left(\bmod 2^{r+3}\right)$. Thus $b_{s}^{2} \neq 1\left(\bmod 2^{s}\right)$.

Lemma 10. If $r$ and $s$ are positive powers of the same odd prime $p$, then $\left|a_{r s}\right|>\left|a_{s}\right|$.

It is sufficient to prove that $\left|a_{p^{t+1}}\right|>\left|a_{p^{t}}\right|$. For $p=3$ or 5 , the lemma is true by Lemma 9 . Now assume $p>5$. Use (6) to get

$$
a_{p^{t+1}}=a_{p^{t}}\left[b_{p^{t}}^{p-1}-(p-2) 2^{p^{t}} b_{p^{t}}^{p-2}+\cdots\right]
$$

all subsequent terms containing $2^{2 p^{t}}$ as a factor. Let $p=k \cdot 2^{u}+1, k$ odd. Then $b^{p-1} \equiv 1\left(\bmod 2^{u+2}\right)$. Now $p^{t} \geqq k \cdot 2^{u}+1$. If $k=1$, then $u>2$ since $p>5$, and $2^{p^{t}}>2^{u+2}$ since $2^{u}+1>u+2$. If $k>1$, then $k \cdot 2^{u}+1$ $>u+2$ for $u \geqq 1$. Thus $\left|a_{p^{t+1}}\right|>\left|a_{p^{t}}\right|$.

LEMMA 11. For $r$ and seach greater than 2 , then $\left|a_{r s}\right|>\left|a_{s}\right|$.

Let $p^{u}$ divide $r s$, but not $s$. If $p$ is $2,3,5$, or 13 , then $\left|a_{r s}\right|>\left|a_{s}\right|$ by Lemmas 8 and 9. If $p$ is some other prime, then $\left|a_{r s}\right| \geqq\left|a_{s}\right|$, and 
$\left|a_{r s}\right| \geqq\left|a_{p^{u}}\right|>1$, by Lemma 7 and [1]. Since $s$ is not a multiple of $p^{u}$, $a_{s}$ is not a multiple of $a_{p^{u}}$, by Lemmas 7 and 10. Were $\left|a_{r s}\right|=\left|a_{s}\right|$ then $a_{8}$ would be a multiple of $a_{p^{u}}$, hence $\left|a_{r s}\right|>\left|a_{s}\right|$.

THEOREM 1. Except for $a_{1}=a_{2}=1, a_{3}=a_{5}=a_{13}=-1$, and for $a_{4}=a_{8}$ $=-3$, if $\left|a_{r}\right|=\left|a_{s}\right|$, then $r=s$.

This is a consequence of Lemmas 7,8 , and 11 .

3. The formula $N(c)$. A formula for $N(c)$ will now be developed through a series of lemmas.

LeMmA 12. For $p$ any odd prime $a_{p} \equiv(-7)^{(p-1) / 2}(\bmod p)$, and $b_{p} \equiv 1(\bmod p)$.

Since $a_{7}=7$, and $b_{7}=-13$, the lemma is true for $p=7$. For $p$ any other odd prime, by (3) and (4),

and

$$
2^{p-1} a_{p}=p-\frac{7 p(p-1)(p-2)}{3 !}+\cdots+(-7)^{(p-1) / 2},
$$

$$
2^{p-1} b_{p}=1-\frac{p(p-1)}{2 !}+\cdots+p(-7)^{(p-1) / 2},
$$

from which the lemma follows by inspection.

LEMmA 13. For $p$ any odd prime, if $(-7 / p)=1$, then $a_{p-1} \equiv 0(\bmod p)$, and if $(-7 / p)=-1$, then $a_{p+1} \equiv 0(\bmod p)$.

If $(-7 / p)=-1$, by Lemma $12,2 a_{p+1}=a_{p}+b_{p} \equiv 0(\bmod p)$. From the two equations $2 a_{p}=a_{p-1}+b_{p-1}$ and $2 b_{p}=-7 a_{p-1}-b_{p-1}$, get $4 a_{p-1}$ $=a_{p}-b_{p}$. Hence for $(-7 / p)=1, a_{p-1} \equiv 0(\bmod p)$.

LEMMA 14. If $p$ is any odd prime, $r>1$, and $a_{s} \equiv 0\left(\bmod p^{r-1}\right)$, then $a_{s p} \equiv 0\left(\bmod p^{r}\right)$.

By (4),

$$
2^{p-1} a_{p s}=a_{s}\left(p b_{s}^{p-1}-\frac{7 p(p-1)(p-2)}{3 !} b_{s}^{p-3} a_{s}^{2}-\cdots\right),
$$

and $a_{s p} \equiv 0\left(\bmod p^{r}\right)$, since the expression in the parentheses has $p$ as a factor of the first term and $a_{s}$ as a factor of every other term.

Lemma 15. For every odd prime $p$ there exists an s such that $a . \equiv 0(\bmod p)$.

For $p=7, s=7$. For $p$ any other odd prime, $s$ is $p-(-7 / p)$, by Lemma 13.

CoROLlaRY. For every odd prime $p$, and for every $r>0$, there exists an $s$ such that $a_{s} \equiv 0\left(\bmod p^{r}\right)$.

This follows from Lemma 14 by mathematical induction. 
We are now ready to derive a formula $N(c)$. Let $q_{i}$ be any odd prime for which -7 is a quadratic residue, and $n_{j}$ any for which -7 is a quadratic non-residue. Let $c$, any positive odd integer, be written in the form

$$
c=7^{0} \prod_{i, j} q_{i}^{e_{i}} n_{j}^{f_{j}}, \quad g \geqq 0, i \geqq 0, j \geqq 0, e_{i} \geqq 1, f_{j} \geqq 1 .
$$

Theorem 2. Let $N(1)=13, N(3)=8$, and for $c$ any odd integer greater than 3 , let $N(c)$ be the least common multiple of all the factors $7^{\circ}$, $q_{i}-1, n_{j}+1, q_{i}^{e_{i}-1}, n_{j}^{f_{j}-1}$, then if $n>N(c),\left|a_{n}\right| \neq c$.

By [1], $\left|a_{n}\right|>1$ if $n>13$. By Theorem 1 , if $n>8\left|a_{n}\right| \neq 3$. By Lemmas $12,13,14$, and $15, a_{N(c)} \equiv 0(\bmod c)$. Suppose $\left|a_{n}\right|=c, c>3$. By Theorem 1, this is true for only one $n$. By Lemma 7 , this $n$ must be a factor of $N(c)$, therefore $n \leqq N(c)$. Thus for all values of $c$, if $n>N(c)$, then $\left|a_{n}\right| \neq c$.

\section{REFERENCES}

1. Th. Skolem, P. Chowla, and D. J. Lewis, The Diophantine equation $2^{n-2}-7=x^{2}$ and related problems, Proc. Amer. Math. Soc. 10 (1959), 663-669.

2. P. Chowla, $A$ class of Diophantine equations, Proc. Nat. Acad. Sci. U.S.A. 45 (1959), 569-570.

UNIVERSITY OF HAWAII

\section{TWO NEW REPRESENTATIONS OF THE PARTITION FUNCTION}

\section{BASIL GORDON}

MacMahon [1] defined a two-rowed partition of the positive integer $n$ as a representation of the form $n=\sum_{i=1}^{r} a_{i}+\sum_{j=1}^{s} b_{j}$ where the $a_{i}$ and $b_{j}$ are positive integers subject to the conditions $r \geqq s, a_{i} \geqq a_{i+1}$, $b_{j} \geqq b_{j+1}, a_{i} \geqq b_{i}$. Such partitions may be conveniently visualized by placing the summands on two rows, the $a_{i}$ on the top row and the $b_{j}$ on the bottom row, with each $b_{i}$ immediately beneath $a_{i}$. Thus for $n=3$ the partitions in question are (omitting + signs)

$$
\begin{aligned}
& 3,21,2,111,11 . \\
& 11
\end{aligned}
$$

In this note the following two theorems will be proved.

THEOREM 1. The number of two-rowed partitions of $n$ satisfying $a_{i}>a_{i+1}, b_{j}>b_{j+1}$ is $p(n)$, the ordinary partition function of $n$.

Received by the editors January 6, 1962 . 